\title{
Laplacian based Image Mosaicing
}

\author{
Sadhana P. Bangarashetti, Pavan N. Kunchur
}

\begin{abstract}
In this paper, we propose to stitch multiple images using the technique of homography estimation. Firstly, Sorting the images, then computing pairwise homography between the images and then by performing image blending the objective is achieved.Then a novel decision fusion framework based on spanning tree and exif data to obtain the order of images is proposed. After the order of images are obtained, to estimate the pairwise projective homographies between the images is done. Then we warp the images according to the computed homography. The warped images later undergo feathering and laplacian blending in order to obtain seamless stitching.

Keywords: Homography,Novel Decision,Laplacian Blending.
\end{abstract}

\section{INTRODUCTION}

The process of panorama stitching is generally seen to be important for both commercial and aesthetic usage. Even though there are devices available for panorama generation, lack of availability of such devices, cost of expensive digital cameras etc.. motivates the need to have synthetic panorama generation. The desire to create full panoramic images is overwhelming and importance of automatic image stitching is quite high. There are different techniques that can be applied to obtain panorama generation. The most common one being Perspective Projection and Cylindrical Projection.In Perspective projection based approach, we propose to compute the perspective projected homography between the images and then try to warp the target images on to a plane. In cylindrical Projection approach, we rely on the results obtained from the image projection on to cylindrical co-ordinate system. The basic idea here is that, if the images are projected onto the cylindrical co-ordinate system, the resultant images are related by an affine transformation rather than the projective transformation.In this assignment, we propose to implement the projective transformation based approach. Here, we first propose to compute the homography and then warp it. The result is then subjected to blending operation to obtain the seamless panorama

Towards this,the following contributions are made:

1) A novel decision fusion framework based on spanning tree and exif data to obtain the order of images.

2) The pairwise homographies are computed using a non-linear based DLT algorithm

Revised Manuscript Received on April 04, 2020.

Sadhana P.Bangarashetti*, Prof, Deprt of ISE,BEC,Bagalkot

Pavan N.Kunchur, Asst.Professor, Dept of CSE,GIT,Belagavi

(C) The Authors. Published by Blue Eyes Intelligence Engineering and Sciences Publication (BEIESP). This is an open access article under the CC BY-NC-ND license (http://creativecommons.org/licenses/by-nc-nd/4.0/) a. SIFT based feature extraction and FLANN based feature matching algorithm is used to provide the point topoint correspondances.

b. The affine homography is calculated wherever the perspective projection is observed to be lower enoughto obtain speed of execution.

3) We propose a composite of Feathering based blending and Laplacian based blending to obtain seamless edges.

4) The proposed algorithm is demonstrated on the provided dataset and also the dataset captured by us.

\section{LITERATURE SURVEY}

Picture sewing has an expansive writing both in PC vision and PC illustrations. Right now, diagram a few calculations that are firmly identified with our work. Intrigued perusers can allude to [1] for more investigations. Maybe the most significant work is Szeliski et al. [12] who delivers a fix based arrangement to refine camera features with fine modification.Nonetheless, there are two restrictions right now: These patches are necessarily the squares equitably taken out from the image and accordingly this technique produces tremendous quantities of patches or highlights and (2) the fix correspondences are worked from the fix habitats which may be of low difference, straight finished and illuminance-delicate, and, subsequently, the assessed movement can be flimsy. Zhou [11] gives another methodology, which in examination with Szeliski et al. replaces the patches with a set number of highlights and applies a vigorous plan dependent on the edge invariance of highlight vectors and the pivot invariance of highlight matches. A BMA dependent on enlightenment standardization (brightening BMA) is additionally acquainted with locate the underlying relocation field to refine highlight coordinating. In any case, the brilliant area search strategy to refine the camera central length is insecure in light of the fact that it needs procedures to use the outcomes from the past layer and can without much of a stretch lead to a nearby least.Another drawback of Chen et al's. work is the brightening standardization step is insecure on account of the approximated reflectance. To beat these impediments, we propose another methodology called TVBMA, where TV-scale pictures with versatile scale designs are embraced to the enlightenment BMA for calculating an even and vertical relocations in the coarsest layer. In contrast to the available sewing considers, we propose two extra strides to refine the movements acquired from the past layer.We adjust it to evacuate low complexity pixels which may be straight finished during the limitation of coordinating highlights.

Published By:

Blue Eyes Intelligence Engineering DOI: 10.35940/ijeat.D7644.049420

Journal Website: www.ijeat.org

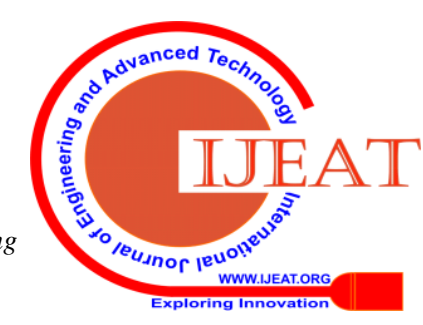


To additionally improve the nature of highlight matches, RANSAC [3], which has been end up being strong to evacuate exceptions $[8,10]$, is likewise fused. Recently, two studies also rely on the invariance properties of feature vectors and matches [10,12]. The method bypasses its difficult settings and instead iteratively finds the variable focal length and traditional parameter.Image bonding based on feature-based techniques is considered an area of active research in computer vision. This section examines some of the previous work that has been done for the feature-based image bonding process. For example, Patel et al. [4] describes the basic methods used to create a panoramic picture and presented some of the basic techniques used in image mosaic. By combining different algorithms and depending on the application, they created new and better image mosaic algorithms. Yanli and Zhenjiang [5] shows a error free image mosaic algorithm in which the author used the SUSAN corner detector to create the characteristic point on epipolar and homography constraints. The straight weighted change strategy is utilized during the time spent picture combination. Bheda and Joshi [11] have examined some regularly utilized strategies for include extraction for picture mosaicing applications. Harris Comer discovery calculation is rotationally invariant and can perform well without scale distinction. SUSAN is additionally a comer location system with a cover that figures the power contrasts to discover the comers, yet this technique isn't scaled invariant. A pivot and scale-invariant calculation called FAST has improved execution time without commotion. Filter, SURF, and PCA-SIFT are descriptor-based calculations and have focal points over various conditions. Filter is invariant to turn, brightening, and relative change changes, and shows great execution in the greater part of the cases however has moderate execution time. SURF is better than execution time. PCASIFT decreased the execution time of SIFT coordinating yet was demonstrated to less compelling than SIFT in removing the element focuses.

\section{PROPOSED METHODOLOGY}

The proposed methodology is demonstrated in Figure 1.

The entire framework can be divided into the following sections:

1. Sorting of images 2. Computing Homography 3. Warping of images 4. Image Blending

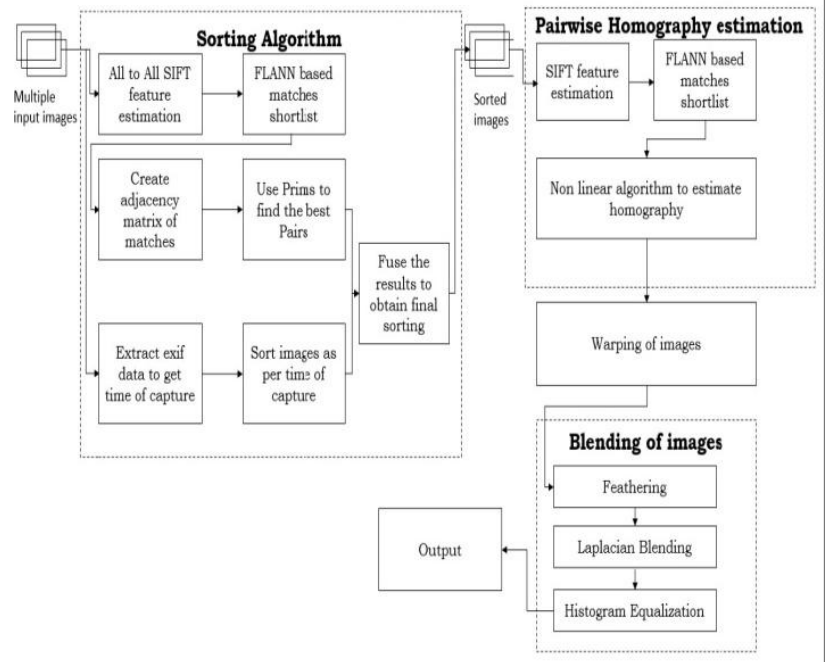

Figure 1: Proposed Framework
Sorting of Images: In this stage, we propose to sort the given images based on the features such that they are suitable for panorama generation. For this,we estimate the SIFT features every images with every other images. Then, we propose to Perform FLANN based matching test to estimate the number of keypoints that match. This data is now used to create an adjacency matrix. We subtract every number of the adjacency matrix with a maximum number so that the adjacency matrix can be used for computation of spanning tree. This gives us the pair wise matching images. The result of pairwise matching results now fused with the data of the time being captured. This is generally done with the intuition that a user usually captures images in an order.

Pairwise Homography estimation: In this stage, we propose to compute homography. For this, we propose to estimate the SIFT features for each of the images with every other images. Then, we propose to Perform FLANN based matching test to estimate the number of keypoints that match. These keypoints are now used to compute the homography matrix using the non linear algorithm available in openCV.

Warping of images: This is a very simple step of multiplying the target image with the computed homography. We propose to choose the centremost picyure as reference image,then propose to warp other images with respect to this. The images to the left are simply warped with the computed homography matrix and the ones to the right are warped with the inverse of it.

Blending of images: We propose blending in two stages. In first stage, we propose to perform feathering to remove the intensity inconsistency and then we perform laplacian blending on that to eliminate the corresponding seams.

\section{RESULTS AND DISCUSSIONS}

The proposed algorithm works well for most of the datasets that have been put-frothed. The result is observed to be stretched in the last image due to the issue in the capture of the dataset.

Results of Sorting of images: For the dataset 1 , the given order of images is 2,3,4,8 and the expected order is $8,2,3,4$. The algorithm is able to predict the same.

Similarly, for the other dataset, the expected order and estimated order is shown below.

Table 1: The estimated order

\begin{tabular}{|l|l|l|}
\hline Dataset & Expected Order & Estimated Order \\
\hline 2 & $4,3,1,2$ & $4,3,1,2$ \\
\hline 3 & $1,4,2,3$ & $1,4,2,3$ \\
\hline 4 & $1,2,3,4$ & $1,2,3,4$ \\
\hline 5 & $2,7,5,1,4,3,6$ & $2,7,5,1,4,3,6$ \\
\hline
\end{tabular}

Fig 2 and Fig 3 shows the results of left warping and right warping shown in respectively. Here first two are the input images and the last one is the output image.

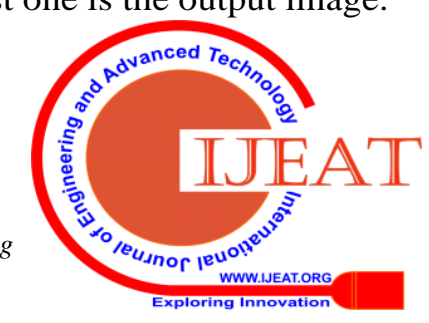



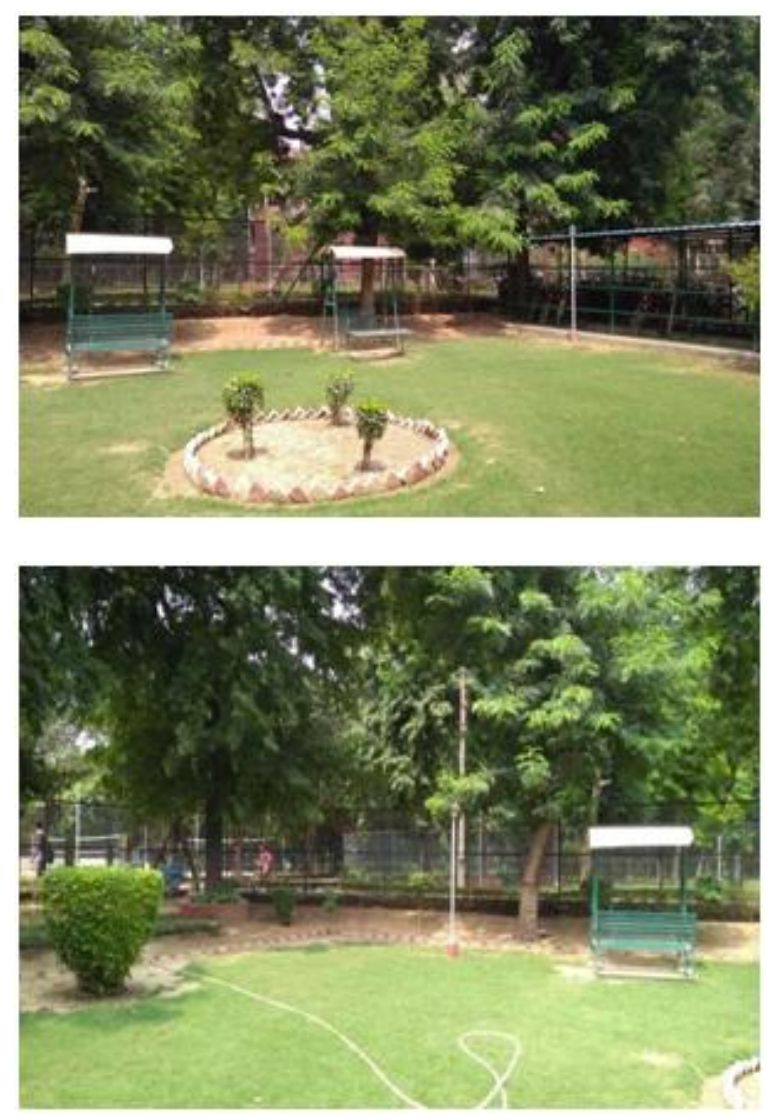

$+$
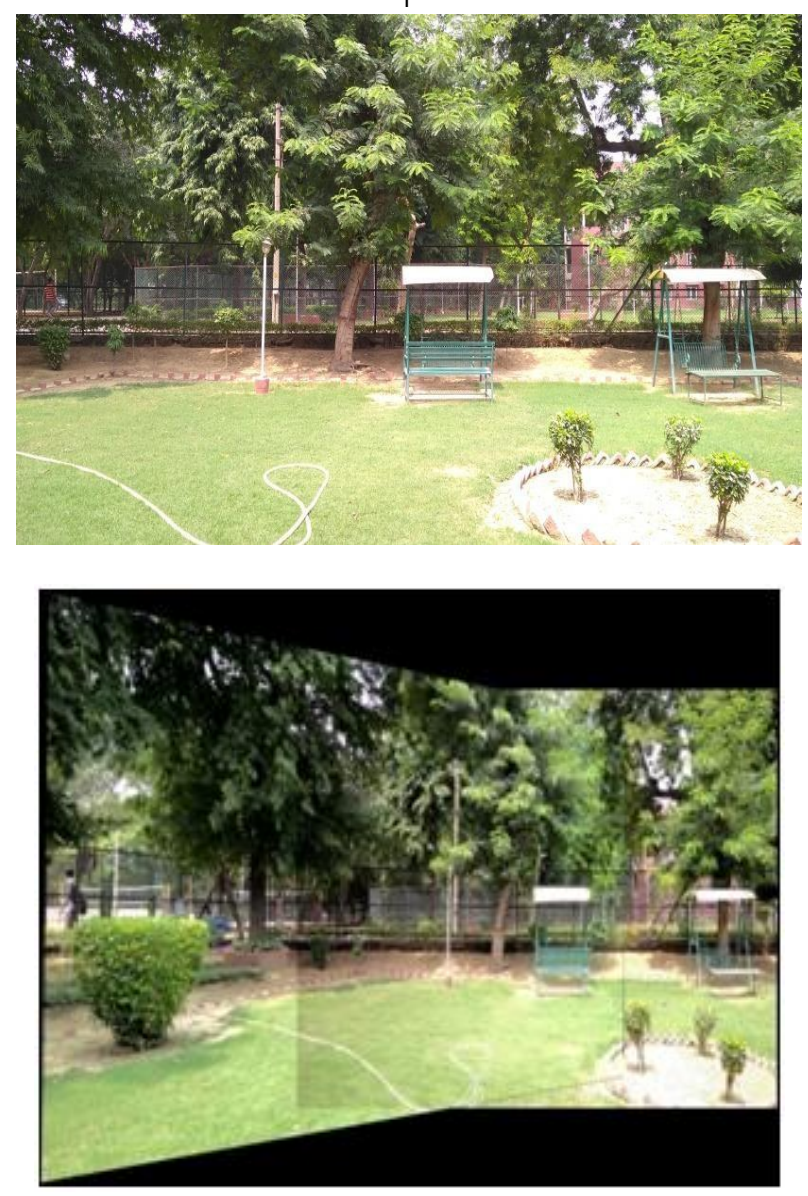

Retrieval Number: D7644049420/2020@BEIESP DOI: 10.35940/ijeat.D7644.049420

Journal Website: www.ijeat.org

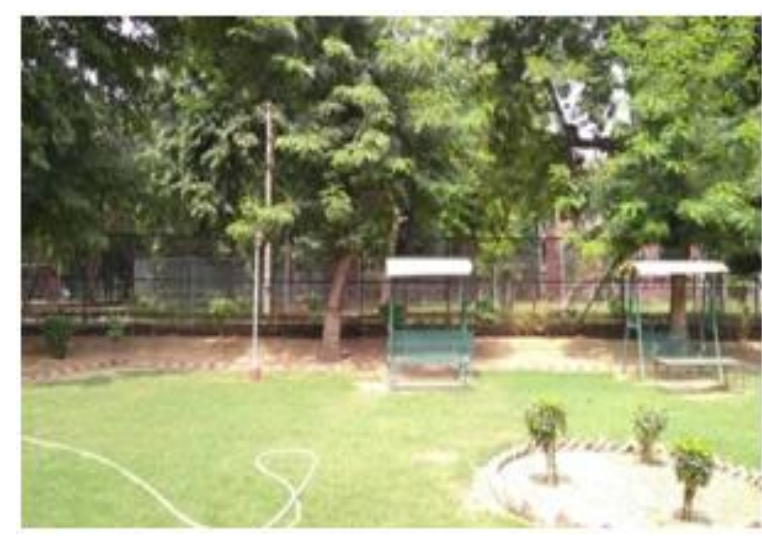

Figure 2:Sample left warping

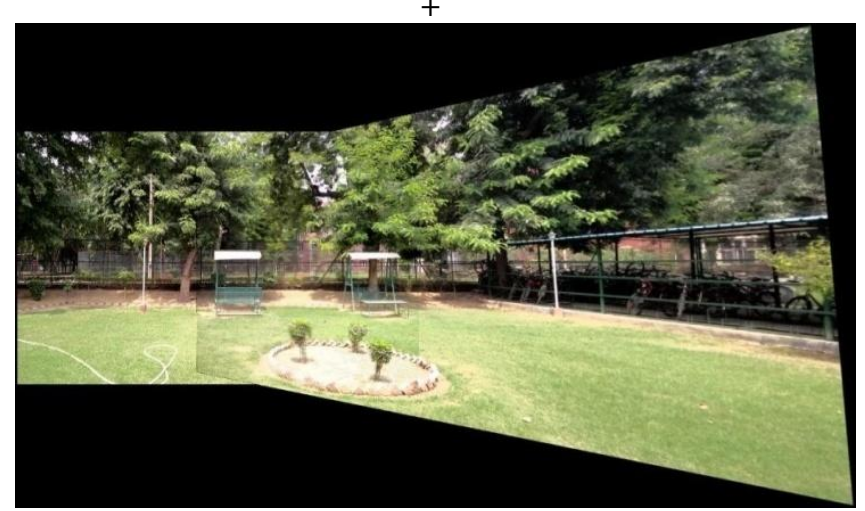

Figure 3: Sample Right Warping

Results of Blending:

Figure 4 and 5 gives the blending.

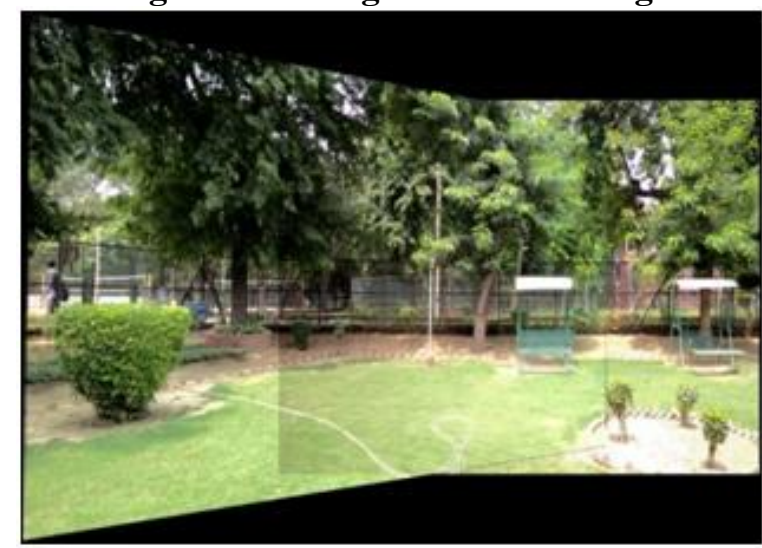

Figure 4: Before Blending

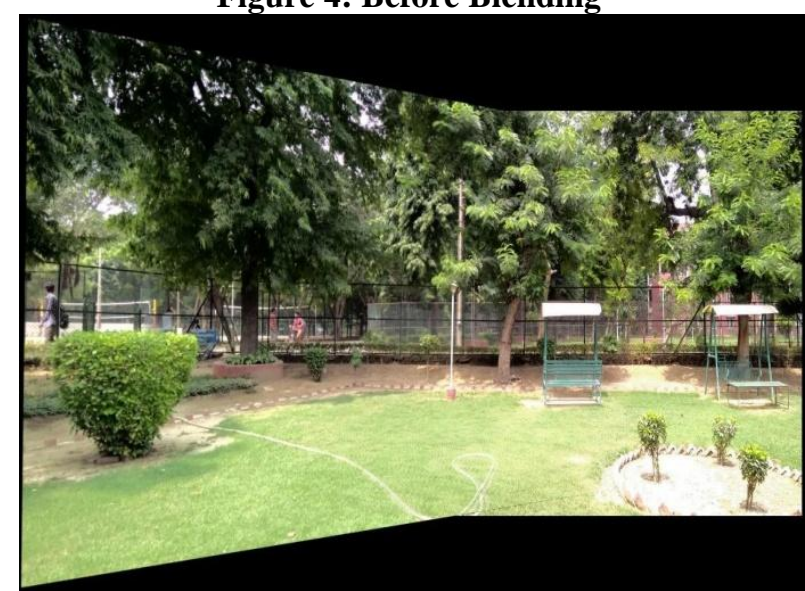

Figure 5: After Blending

Published By:

Blue Eyes Intelligence Engineering

\& Sciences Publication

(C) Copyright: All rights reserved.

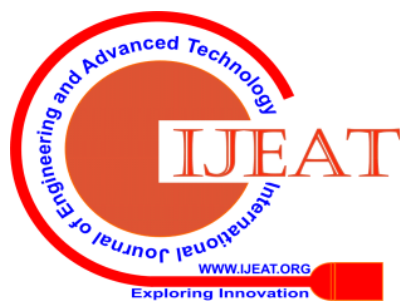




\section{Laplacian based Image Mosaicing}

Result of the Complete Pipeline:

The images in Figure 6 show the results of the complete pipeline. The first three are the input images and the last one is the output image.
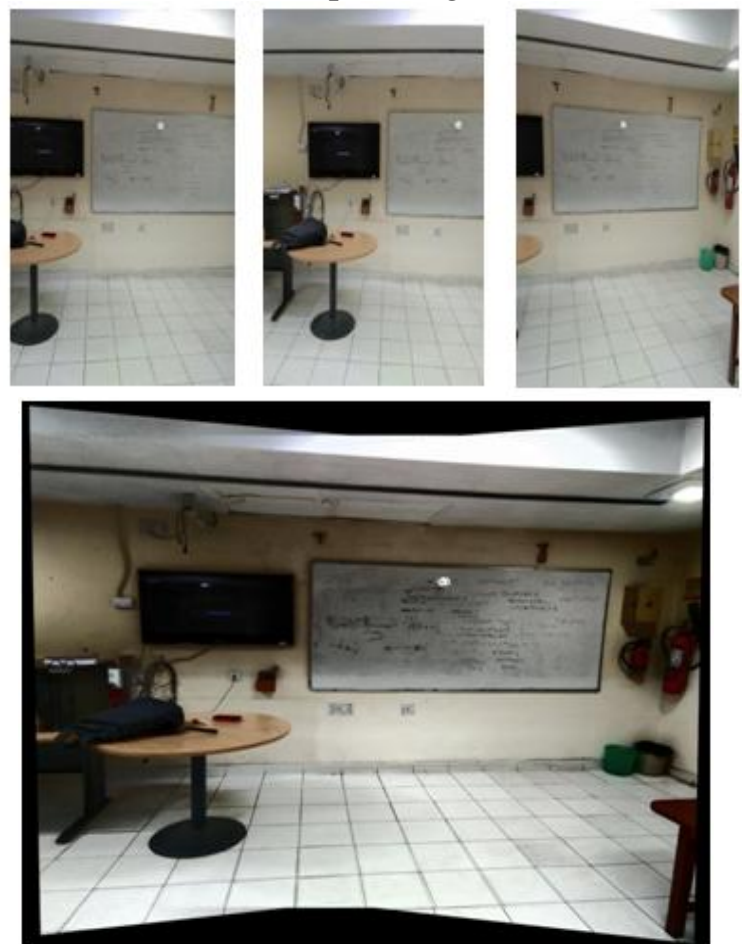

Figure 6: Result of the Complete Pipeline

\section{CONCLUSION}

In this paper, we have proposed to stitch multiple images using the technique of homography estimation. We achieved the proposed objective by first sorting the images, then computing pairwise homography between the images and then by performing image blending. We also proposed a novel decision fusion framework based on spanning tree and exif data to obtain the order of images. The present pipeline works well for the purpose of panorama stitching.

\section{REFERENCES}

1. M. Mirmehdi, P. Clark, and J. Lam. Extracting low resolution text with an active camera for OCR. In Proc. IX Spanish Sym. Pat. Rec. and Image Proc., pages 43-48, May 2001.

2. T. Nakao, A. Kashitani, and A. Kaneyoshi. Scanning a document with a small camera attached to a mouse. In Proc.WACV'98, pages 63-68, 2015.

3. B. S. Reddy and B. N. Chatterji. An FFT-based technique for translation, rotation, and scale-invariant image registration.IEEE Trans. Image Proc., 5(8):1266-1271, 2014.

4. K. Schutte and A. M. Vossepoel. Accurate mosaicking of scanned maps, or how to generate a virtual a0 scanner. In Proc. ASCI'95, pages 353-359, 2015.

5. A. P. Whichello and H. Yan. Document image mosaicing. In Proc. ICPR, pages 1081-1083, 2013.

6. A. Zappala, A. Gee, and M. J. Taylor. Document mosaicing. Image and Vision Computing, 17(8):585-595, 2012

7. M. Trajkovic and M. Hedley, "Fast comer detection," Image Vision Computer, Vol. 16, no. 2, pp. 75-87,1998.

8. Gonzalez R.C and Wintz P, Digital Image Processing, New York, Academic, 2014

9. William M. Newman and Robert F. Sproull, Principles of Znkractive Computer Graphics, McGraw-Hill, 2017.

10. Ani1 K. Jain, Fundamentals of Digital ImageProcessing, PrenticeHall, 2014

11. P. Dani S. Chaudhuri. Automated assembling of images: Image montage preparation. Pattern Recognition, 28(3):431-445, 2016
12. Waheeda Dhokley, Mandar Sohani, Dr.S.A.Patekar,'Image Mosaicing using Enhance Dynamic Programming Based Stitching Technique",in International conference \& Workshop on Recent Trends \& Technology(TCET),2015,Page 1-5.

\section{AUTHOR PROFILE}

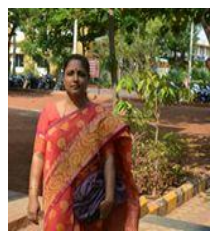

I,Dr. Sadhana P. Bangarashetti currently am working as professor in the department of Information Science and Engg. , Basaveshwar Engg. College, Bagalkot. My main research areas are Image Processing, Big Data Analytics and Internet of Things. I have authored over 8 journal and conference papers and provided my service as reviewer for the first IEEE international conference on Advances in Information Technology (ICAIT2019) held at department of Computer Science and Engg. , Adichunchanageri Institute of Technology, Chikkamagaluru, Karnataka, India. I am currently Advisory Committee Member for the ICSET ' 20 Conference organized by Institute for Engineering Research and Publication, Pune, Maharashtra.

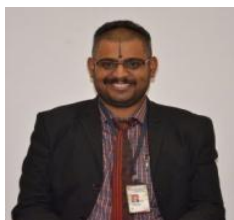

I,Pavan N.Kunchur,Currently serving as Asst.Prof in department of computer science and engineering at Gogte Institute of Technology,Belagavi,Karnataka,Pursuing Ph.D in Image Processing.8 years of teaching experience and also interested in Data Structures,Networking.

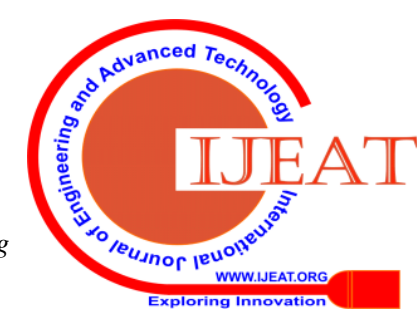

